\title{
Measurement of Gamma Radiation in an Automobile Mechanic Village in Abuja, North Central, Nigeria
}

\author{
${ }^{* 1}$ IU; JAMES, IF; MOSES, JN; VANDI \\ Nuclear Technology Centre, Nigeria Atomic Energy Commission, Sheda-Abuja, P.M.B O7, Gwagwalada, Abuja \\ Email: jamesfam2002@yahoo.com
}

KEYWORDS: Radiation Measurement, Apo Automobile Mechanic Village, Background Radiation, Dose Equivalent, Annual Effective Dose, Automobile Technicians

\begin{abstract}
Environmental radiation measurement was carried out in an automobile mechanic village, Apo, Abuja, Nigeria. An in-situ measurement approach was adopted using RDS-200 Universal Survey Meter and a handheld Global Positioning System (Garmin GPS 76S) equipment. It was observed that the dose equivalent varied from $0.04 \mu \mathrm{Sv} / \mathrm{h}$ to $0.22 \mu \mathrm{Sv} / \mathrm{h}$ with a mean of $0.10 \pm 0.03 \mu \mathrm{Sv} / \mathrm{h}$ which is below the standard background radiation of $0.133 \mu \mathrm{Sv} / \mathrm{h}$ The study also revealed that the average annual effective dose rate is approximately $0.20 \pm 0.06 \mathrm{mSv} / \mathrm{yr}$ which is lower than the value of $1.0 \mathrm{mSv} / \mathrm{yr}$ averaged over five consecutive years according to the dose limit placed by the Basic Safety Standards (BSS) SCHEDULE II and the International Commission on Radiological Protection (ICRP) REPORT 60. This indicates that the automobile technicians, craftsmen and the people living and working within the area are safe and are not exposed to high doses of radiation as a result of activities in the Apo Automobile Mechanic Village. @ JASEM
\end{abstract}

\section{http://dx.doi.org/10.4314/jasem.v18 i2.21}

Introduction: Radiation, because of the adverse health effect when persons are over exposed to ionizing radiation, is feared by many people worldwide and Nigerians are no exemption (Oyeyinka et al., 2012). The earth's atmosphere especially the human populace is exposed to both non-ionizing and ionizing radiation from different sources, which include natural and artificial sources. Prominent among the natural sources are the primordial radionuclides $\left({ }^{238} \mathrm{U}\right.$ and ${ }^{232} \mathrm{Th}$ and their progenies, and ${ }^{40} \mathrm{~K}$ ), while the artificial sources include, anthropogenic radionuclide such as ${ }^{137} \mathrm{Cs}$, ${ }^{90}$ Sr, etc. (Avwiri et al., 2010).

The basic difference between ionizing radiation and other common types of radiation in the environment such as heat is that it possesses sufficient energy to cause ionization. In water of which cell are largely composed, ionization can lead to molecular changes and to the formation of chemical species of a type which are damaging to the chromosome material. Ionizing radiation injury is dependent on a number of factors including: The nature (alpha $(\alpha)$, beta $(\beta)$, and gamma $(\gamma)$ ) and energy of the radiation, the dose, time of exposure, homogeneity of dose and shielding (ICRP, 1991). When the dose and dose rate is within the accepted level, the effect of radiation is small and most time no effect is noticed, although the effect of low level radiation are not yet completely understood (Olarinoye et al., 2010). Human body is permanently irradiated from two ionizing radiation sources: External and Internal. External radiation sources can either be natural (cosmic, Terrestrial) or artificial
(Medical, Commercial and Industrial sources), both of equal risk to man. Small traces of many naturally occurring radioactive materials are present inside the human body. These come mainly from naturally occurring radioactive nuclides present in the food we eat and in the air we breathe. These isotopes include tritium $\left({ }^{3} \mathrm{H}\right)$, carbon-14 $\left({ }^{14} \mathrm{C}\right)$, and potassium-40 $\left({ }^{40} \mathrm{~K}\right)$ (Oyeyinka et al., 2012). The level of the natural radioactivity in the soil and in the surrounding environment as well as the associated external exposure due to the gamma radiation depends primarily on the geological and geographical conditions of the region (UNSCEAR, 2000). The geological and geographical definition of an environment dictate to a good degree the radionuclides contained in the soil and rocks there (Tchokossa et al., 2012). Soil contains small quantities of radioactive elements along with their progeny (Olarinoye et al., 2010). However, since radiation is known to cause cancer, it is prudent to monitor the environment for radioactivity (MDH 2008).

Environmental Radiation Monitoring is a systematic collection and analysis of certain environmental media such as air, milk and water to determine the level of radioactivity present, in which various levels of radioactivity are compared with safety standards to ensure a safe environment. This is introduced so as to protect the public and the environment from hazards associated with ionizing radiation (MDH 2008).

An in-situ measurement of the background radiation level was carried out at the vicinity of three campuses 
of two major tertiary institutions in Minna, Niger State. The results of the investigation revealed that the average annual effective dose obtained is 0.189 mSv/annum (Olarinoye et al., 2010). A study of the background radiation in Akwanga, Nasarawa State showed that the annual mean equivalent doses for indoor and outdoor backgrounds are 1.29 \pm 0.13 and $0.31 \pm 0.14 \mathrm{mSv} / \mathrm{yr}$ respectively (Sadiq and Agba, 2011). In Abuja, Estimation of Radiation Dose Rate Levels was carried out around a Nuclear Establishment in Abuja, North Central, Nigeria. It was observed that the dose equivalent rate varied from $0.106 \pm 0.032$ to $0.212 \pm 0.036 \mu \mathrm{Sv} / \mathrm{h}$ with a mean of $0.149 \pm 0.032 \mu \mathrm{Sv} / \mathrm{h}$. These results though slightly above the standard background radiation of $0.133 \mu \mathrm{Sv} / \mathrm{h}$, they are below the ICRP maximum permissible limit of $0.57 \mu \mathrm{Sv} / \mathrm{h}$ and may not pose any danger to the radiation workers, the general public and the environment (Oyeyinka et al.,2012). Determination of Absorbed and Effective Dose from Natural Background Radiation around a Nuclear Research Facility was carried out in Zaria (Mohammad et al., 2011). It was observed that the estimated total annual effective dose outdoor for the sites range from 27.3-79.9 $\mu \mathrm{Sv} \mathrm{y}^{-1}$. A Measurement of Gamma Radiation in Automobile Mechanic Workshops was carried out in an area of Benin City, Nigeria (Nworgu et al., 2012). The study revealed that the average annual effective dose rate from these sites is approximately $0.40 \mathrm{mSv} / \mathrm{yr}$ which is lower than the value of $1.0 \mathrm{mSv} / \mathrm{yr}$ averaged over five consecutive years according to the dose limit placed by the Basic Safety Standards (BSS) SCHEDULE II and the International Commission on Radiological Protection (ICRP) REPORT 60. However, the external background ionizing radiation exposure within the sites investigated varied between 0.1272 and $0.01411 \mathrm{mR} / \mathrm{hr}$ with an average of $0.01314 \pm$ $0.000658 \mathrm{mR} / \mathrm{hr}$ in the locality which is relatively higher than the standard background radiation of $0.011 \mathrm{mR} / \mathrm{hr}$ recommended by the US Nuclear Radiation Commission. This level of background radiation seems to suggest that there is a possibility of the existence of radio nuclides within the area. Also the natural background radiation dose/dose rate has been investigated by many researchers in other parts of the world and a wide range of results are reported (Amiri et al., 2011). On the relevance of radiation monitoring, the Office of Radiation Protection (Office of Radiation Protection, 2008) stated that through radiation monitoring, sample collection and data analysis, the environment is protected from hazards associated with ionizing radiation.

There are more than $1,577,000$ metric tons of irradiated scrap metals available and these metals come from decommissioned nuclear reactors and nuclear weapons, tons of steel from buildings that contain radioactivity which are part of the 'hot metal" scrap being introduced for recycling (Howdershelt,
2000). Radioactive metals like gold, silver, carbon steel, stainless steel, aluminium, nickel and copper are being made available for recycling (Howdershelt, 2000). Scrap metal can contain sources of radiation with the associated environmental and health risks. Higher levels of radiation are possible and may stem from losses, accidents or the inadvertent disposal of radioactive material (Lenka and Peter, 2010).

In Nigeria, automobile mechanic workshops are located or concentrated in areas known as mechanic villages. These places are officially designated for repairs and servicing of motor vehicles (Angela et al., 2011). Automobile Mechanic workshops in most cities in Nigeria could pose serious health hazards to the auto technicians (locally called mechanics). This is as a result of the fact that these mechanics are exposed to metals indiscriminately dumped in the vicinity of the workshop that might have been contaminated by other radioactive metals in the course of production (Nworgu et al., 2012).

The proper monitoring and evaluation of the radiation emanating from automobile mechanic workshops in order to provide accurate data as part of environmental monitoring research for proper assessment of radiation exposure rate in Abuja motivated this study.

\section{MATERIALS AND METHODS}

Apo Automobile Mechanic village is an industrial/mechanic town located in the suburb of Nigeria's Federal Capital Territory, Abuja. It is known for trade and services in various aspect of motor vehicle. It prides itself as a home of local technology with a cesspool of mechanic activities such as car servicing and all kinds of repairs, motor battery works, panel beating e.t.c.

Abuja, Nigeria's new capital city is located in the middle of the country. The Federal Capital Territory has a land area of 8,000 square kilometres, which is two and halftimes the size of Lagos, the former capital of Nigeria. The FCT is bounded on the north by Kaduna State, on the west by Niger State, on the east and south-east by Plateau State, and on the southwest by Kogi State.'.

The study was conducted in April, 2013 in and around Apo Automobile Mechanic Village in Abuja, Nigeria. The study area lie within latitude $08^{0} 57^{\mathrm{N}}$ and $08^{\circ} 58^{\top} \mathrm{N}$ and longitude $07^{0} 29^{\prime} \mathrm{E}$. An in-situ approach of background radiation measurement was adopted and preferred to enable samples maintain their original environmental characteristics. A universal survey meter, RDS-200 [figure 1] and a Geographical Positioning System (GPS) Garmin 76S [figure 2] were used. The RDS-200 Universal Survey Meter is an excellent, portable multipurpose radiation meter for a wide range of applications. It is especially 
designed for situations where accurate measurements at low dose rate levels are of importance. The meter has an interface for the external gamma probes GMP$12 \mathrm{H} / 12 \mathrm{~L}$ or beta/contamination measurement probe GMP-11/15. A connector for the attachment of the meter to a PC is located at the bottom part of the meter and is equipped with protective cover. The RDS-200 utilizes field-proven measurement electronics and can also be used as a local display unit with the RADOS AAM-90 Area Monitoring System. The meter measures $\gamma$-radiation and beta radiation with an external probe detector It also measures equivalent dose rate within $0.05 \mu \mathrm{Sv} / \mathrm{hr}-10$

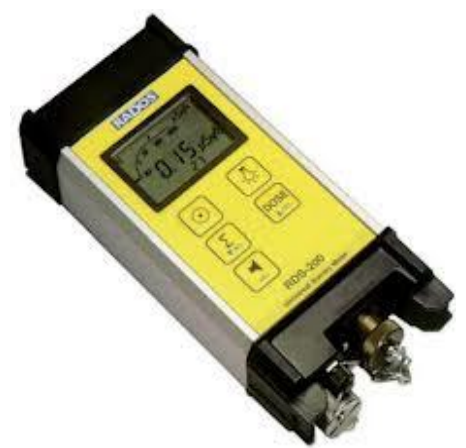

Fig 1: RDS-200 Universal Survey Meter $\mu \mathrm{Sv} / \mathrm{hr}$. The monitor was suspended in air at one meter above the ground level. Readings were obtained between the hours of 1200 and 1600 hours since the exposure rate meter has a maximum response to environmental radiation within these hours. Three readings were taken in each location and the average calculated.

The equation below is used to calculate the annual effective dose rate in milli-sievert per year $(\mathrm{mSv} / \mathrm{y})$. Annual effective dose rate $(\mathrm{mSv} / \mathrm{yr})=$ Equivalent dose rate $(\mu \mathrm{Sv} / \mathrm{hr}) \times 8760 \mathrm{~h} / \mathrm{yr} \times 0.2$ (occupancy factor) $\times 10^{-3}(1)$ (Tayyeb et. al., 2012)

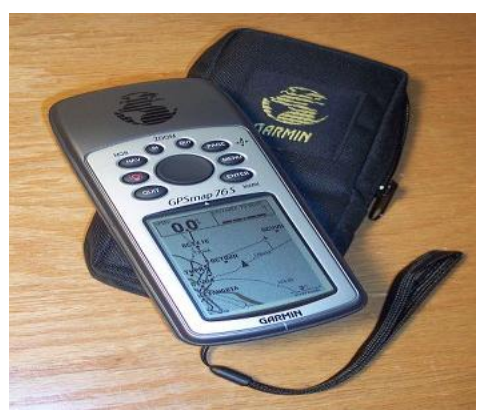

Fig 2: GPS Garmin 76S

\section{RESULTS AND DISCUSSION}

Table 1 shows the average equivalent dose rate and the annual effective dose rate. A total of 133 measurements were taken within and around the Apo Mechanic Village. Figure 3 shows the average equivalent dose rate of different location. Generally, from the result in Figure 3 the average equivalent dose rate ranged between $0.04 \mu \mathrm{Sv} / \mathrm{hr}$ to $0.22 \mu \mathrm{Sv} / \mathrm{hr}$ with a mean of $0.10 \pm 0.03 \mu \mathrm{Sv} / \mathrm{hr}$ which is below the standard background radiation of $0.133 \mu \mathrm{Sv} / \mathrm{hr}$. Of all the locations AMV013 had the highest dose rate value while AMV072 and AMV120 had the least dose rate value.

Table 1: Average Equivalent dose rate and annual effective dose rate of different locations measured

\begin{tabular}{|c|c|c|c|c|}
\hline \multirow[t]{2}{*}{ Site Id } & \multicolumn{2}{|c|}{ Location } & \multirow{2}{*}{$\begin{array}{c}\text { Average } \\
\text { Equivalent } \\
\text { Dose Rate } \\
\left(\mu \mathrm{Svhr}^{-1}\right)\end{array}$} & \multirow{2}{*}{$\begin{array}{l}\text { Annual } \\
\text { Effective } \\
\text { Dose Rate } \\
\left(\mathrm{mSvyr}^{-1}\right)\end{array}$} \\
\hline & Latitude & Longitude & & \\
\hline AMV001 & $08^{0} 57^{\prime} 304^{\prime \prime} \mathrm{N}$ & $07^{0} 29^{\prime} 864^{\prime \prime} \mathrm{E}$ & 0.10 & 0.18 \\
\hline AMV002 & $08^{0} 57^{\prime} 305^{\prime} \mathrm{N}$ & $07^{0} 29^{\prime} 876^{\prime \prime} \mathrm{E}$ & 0.05 & 0.09 \\
\hline AMV003 & $08^{0} 57^{\prime} 286^{\prime} \mathrm{N}$ & $07^{0} 29^{\prime} 902^{\prime \prime} \mathrm{E}$ & 0.15 & 0.26 \\
\hline AMV004 & $08^{\circ} 57^{\prime} 776^{\prime \prime} \mathrm{N}$ & $07^{0} 29^{\prime} 898^{\prime \prime} \mathrm{E}$ & 0.14 & 0.25 \\
\hline AMV005 & $08^{0} 57^{\prime} 271^{\prime \prime N}$ & $07^{0} 29^{\prime} 878^{\prime \prime} \mathrm{E}$ & 0.09 & 0.16 \\
\hline AMV006 & $08^{0} 57^{\prime} 277^{\prime} \mathrm{N}$ & $07^{0} 29^{\prime} 862^{\prime \prime} \mathrm{E}$ & 0.13 & 0.23 \\
\hline AMV007 & $08^{0} 57^{\prime} 263^{\prime} \mathrm{N}$ & $07^{0} 29^{\prime} 856^{\prime \prime} \mathrm{E}$ & 0.07 & 0.12 \\
\hline AMV008 & $08^{0} 57^{\prime} 291^{\prime \prime N}$ & $07^{0} 29^{\prime} 846^{\prime \prime} \mathrm{E}$ & 0.13 & 0.23 \\
\hline AMV009 & $08^{0} 57^{\prime} 290^{\prime \prime} \mathrm{N}$ & $07^{0} 29^{\prime} 833^{\prime \prime} \mathrm{E}$ & 0.12 & 0.21 \\
\hline AMV010 & $08^{0} 57^{\prime} 281^{\prime \prime} \mathrm{N}$ & $07^{0} 29^{\prime} 821^{\prime \prime} \mathrm{E}$ & 0.11 & 0.19 \\
\hline AMV011 & $08^{0} 57^{\prime} 313^{\prime} \mathrm{N}$ & $07^{0} 29^{\prime} 823^{\prime \prime} \mathrm{E}$ & 0.12 & 0.21 \\
\hline AMV012 & $08^{0} 57^{\prime} 341^{\prime \prime N}$ & $07^{0} 29^{\prime} 836^{\prime \prime} \mathrm{E}$ & 0.18 & 0.32 \\
\hline AMV013 & $08^{0} 57^{\prime} 345^{\prime} \mathrm{N}$ & $07^{0} 29^{\prime} 847^{\prime \prime} \mathrm{E}$ & 0.22 & 0.39 \\
\hline AMV014 & $08^{0} 57^{\prime} 341^{\prime \prime} \mathrm{N}$ & $07^{0} 29^{\prime} 851^{\prime \prime} \mathrm{E}$ & 0.13 & 0.23 \\
\hline AMV015 & $08^{0} 57^{\prime} 352^{\prime \prime} \mathrm{N}$ & $07^{0} 29^{\prime} 856^{\prime \prime} \mathrm{E}$ & 0.08 & 0.14 \\
\hline AMV016 & $08^{0} 57^{\prime} 419^{\prime} \mathrm{N}$ & $07^{0} 29^{\prime} 880^{\prime \prime} \mathrm{E}$ & 0.12 & 0.21 \\
\hline AMV017 & $08^{0} 57^{\prime} 032^{\prime \prime} \mathrm{N}$ & $07^{0} 29^{\prime} 894^{\prime \prime} \mathrm{E}$ & 0.10 & 0.18 \\
\hline AMV018 & $08^{0} 57^{\prime} 400^{\prime} \mathrm{N}$ & $07^{0} 29^{\prime} 900^{\prime \prime} \mathrm{E}$ & 0.13 & 0.23 \\
\hline AMV019 & $08^{0} 57^{\prime} 399^{\prime} \mathrm{N}$ & $07^{0} 29^{\prime} 807^{\prime \prime} \mathrm{E}$ & 0.12 & 0.21 \\
\hline AMV020 & $08^{0} 57^{\prime} 391^{\prime \prime N}$ & $07^{0} 29^{\prime} 901^{\prime \prime} \mathrm{E}$ & 0.09 & 0.16 \\
\hline AMV021 & $08^{0} 57^{\prime} 384^{\prime \prime} \mathrm{N}$ & $07^{0} 29^{\prime} 898^{\prime \prime} \mathrm{E}$ & 0.09 & 0.16 \\
\hline AMV022 & $08^{0} 57^{\prime} 388^{\prime \prime} \mathrm{N}$ & $07^{0} 29^{\prime} 908^{\prime \prime} \mathrm{E}$ & 0.07 & 0.12 \\
\hline AMV023 & $08^{0} 57^{\prime} 401^{\prime \prime} \mathrm{N}$ & $07^{0} 29^{\prime} 911^{\prime \prime} \mathrm{E}$ & 0.07 & 0.12 \\
\hline AMV024 & $08^{0} 57^{\prime} 406^{\prime} \mathrm{N}$ & $07^{0} 29^{\prime} 866^{\prime \prime} \mathrm{E}$ & 0.07 & 0.12 \\
\hline AMV025 & $08^{0} 57^{\prime} 395^{\prime} \mathrm{N}$ & $07^{0} 29^{\prime} 870^{\prime \prime} \mathrm{E}$ & 0.14 & 0.25 \\
\hline
\end{tabular}




\begin{tabular}{|c|c|c|c|c|}
\hline AMV026 & $08^{0} 57^{\prime} 387^{\prime \prime} \mathrm{N}$ & $07^{0} 29^{\prime} 874^{\prime \prime} \mathrm{E}$ & 0.10 & 0.18 \\
\hline AMV027 & $08^{0} 57^{\prime} 387^{\prime \prime} \mathrm{N}$ & $07^{0} 29^{\prime} 873^{\prime \prime} \mathrm{E}$ & 0.09 & 0.16 \\
\hline AMV028 & $08^{0} 57^{\prime} 382^{\prime \prime} \mathrm{N}$ & $07^{0} 29^{\prime} 876^{\prime \prime} \mathrm{E}$ & 0.09 & 0.16 \\
\hline AMV029 & $08^{0} 57^{\prime} 270^{\prime \prime} \mathrm{N}$ & $07^{0} 29^{\prime} 870$ ' $\mathrm{E}$ & 0.11 & 0.19 \\
\hline AMV030 & $08^{0} 57^{\prime} 363^{\prime \prime} \mathrm{N}$ & $07^{0} 29^{\prime} 869^{\prime \prime} \mathrm{E}$ & 0.15 & 0.26 \\
\hline AMV031 & $08^{0} 57^{\prime} 371^{\prime \prime} \mathrm{N}$ & $07^{0} 29^{\prime} 851^{\prime \prime} \mathrm{E}$ & 0.17 & 0.30 \\
\hline AMV032 & $08^{0} 57^{\prime} 372^{\prime \prime} \mathrm{N}$ & $07^{0} 29^{\prime} 842^{\prime \prime} \mathrm{E}$ & 0.13 & 0.23 \\
\hline AMV033 & $08^{0} 57^{\prime} 379^{\prime \prime} \mathrm{N}$ & $07^{0} 29^{\prime} 845^{\prime \prime} \mathrm{E}$ & 0.08 & 0.14 \\
\hline AMV034 & $08^{0} 57^{\prime} 377^{\prime \prime} \mathrm{N}$ & $07^{0} 29^{\prime} 845^{\prime \prime} \mathrm{E}$ & 0.06 & 0.11 \\
\hline AMV035 & $08^{0} 57^{\prime} 372^{\prime \prime} \mathrm{N}$ & $07^{0} 29^{\prime} 823^{\prime \prime} \mathrm{E}$ & 0.09 & 0.16 \\
\hline AMV036 & $08^{0} 57^{\prime} 370^{\prime \prime} \mathrm{N}$ & $07^{0} 29^{\prime} 831^{\prime \prime} \mathrm{E}$ & 0.09 & 0.16 \\
\hline AMV037 & $08^{0} 57^{\prime} 384^{\prime \prime} \mathrm{N}$ & $07^{0} 29$ '818' E & 0.11 & 0.19 \\
\hline AMV038 & $08^{0} 57^{\prime} 408^{\prime \prime} \mathrm{N}$ & $07^{0} 29^{\prime} 819^{\prime \prime} \mathrm{E}$ & 0.11 & 0.19 \\
\hline AMV039 & $08^{0} 57^{\prime} 408^{\prime \prime} \mathrm{N}$ & $07^{0} 29^{\prime} 818^{\prime \prime} \mathrm{E}$ & 0.08 & 0.14 \\
\hline AMV040 & $08^{0} 57^{\prime} 411^{\prime \prime} \mathrm{N}$ & $07^{0} 29^{\prime} 831^{\prime \prime} \mathrm{E}$ & 0.10 & 0.18 \\
\hline AMV041 & $08^{0} 57^{\prime} 448^{\prime \prime} \mathrm{N}$ & $07^{0} 29^{\prime} 810$ ''E & 0.07 & 0.12 \\
\hline AMV042 & $08^{0} 57^{\prime} 456^{\prime \prime} \mathrm{N}$ & $07^{0} 29^{\prime} 812^{\prime \prime} \mathrm{E}$ & 0.06 & 0.11 \\
\hline AMV043 & $08^{0} 57^{\prime} 455^{\prime \prime} \mathrm{N}$ & $07^{0} 29^{\prime} 822^{\prime \prime} \mathrm{E}$ & 0.05 & 0.09 \\
\hline AMV044 & $08^{0} 57^{\prime} 453^{\prime \prime} \mathrm{N}$ & $07^{0} 29^{\prime} 830$ ''E & 0.09 & 0.16 \\
\hline AMV045 & $08^{0} 57^{\prime} 449^{\prime \prime} \mathrm{N}$ & $07^{0} 29^{\prime} 830^{\prime \prime} \mathrm{E}$ & 0.12 & 0.21 \\
\hline AMV046 & $08^{0} 57^{\prime} 450^{\prime \prime} \mathrm{N}$ & $07^{0} 29^{\prime} 839^{\prime \prime} \mathrm{E}$ & 0.10 & 0.18 \\
\hline AMV047 & $08^{0} 57^{\prime} 456^{\prime \prime} \mathrm{N}$ & $07^{0} 29^{\prime} 843$ ''E & 0.07 & 0.12 \\
\hline AMV048 & $08^{0} 57^{\prime} 453^{\prime \prime} \mathrm{N}$ & $07^{0} 29^{\prime} 866^{\prime \prime} \mathrm{E}$ & 0.08 & 0.14 \\
\hline AMV049 & $08^{0} 57^{\prime} 458^{\prime \prime} \mathrm{N}$ & $07^{0} 29^{\prime} 866^{\prime \prime} \mathrm{E}$ & 0.08 & 0.14 \\
\hline AMV050 & $08^{0} 57^{\prime} 472^{\prime \prime} \mathrm{N}$ & $07^{0} 29^{\prime} 866^{\prime \prime} \mathrm{E}$ & 0.09 & 0.16 \\
\hline AMV051 & $08^{0} 57^{\prime} 478^{\prime \prime} \mathrm{N}$ & $07^{0} 29^{\prime} 854^{\prime \prime} \mathrm{E}$ & 0.08 & 0.14 \\
\hline AMV052 & $08^{0} 57^{\prime} 473^{\prime \prime} \mathrm{N}$ & $07^{0} 29^{\prime} 850$ '' $\mathrm{E}$ & 0.10 & 0.18 \\
\hline AMV053 & $08^{0} 57^{\prime} 464^{\prime \prime} \mathrm{N}$ & $07^{0} 29^{\prime} 836^{\prime \prime} \mathrm{E}$ & 0.11 & 0.19 \\
\hline AMV054 & $08^{0} 57^{\prime} 475^{\prime \prime} \mathrm{N}$ & $07^{0} 29^{\prime} 827^{\prime \prime} \mathrm{E}$ & 0.12 & 0.21 \\
\hline AMV055 & $08^{0} 57^{\prime} 481^{\prime \prime} \mathrm{N}$ & $07^{0} 29^{\prime} 828^{\prime \prime} \mathrm{E}$ & 0.11 & 0.19 \\
\hline AMV056 & $08^{0} 57^{\prime} 484^{\prime \prime} \mathrm{N}$ & $07^{0} 29^{\prime} 837^{\prime \prime} \mathrm{E}$ & 0.10 & 0.18 \\
\hline AMV057 & $08^{0} 57^{\prime} 497^{\prime \prime} \mathrm{N}$ & $07^{0} 29^{\prime} 818^{\prime \prime} \mathrm{E}$ & 0.07 & 0.12 \\
\hline AMV058 & $08^{0} 57^{\prime} 496^{\prime \prime} \mathrm{N}$ & $07^{0} 29^{\prime} 828^{\prime \prime} \mathrm{E}$ & 0.07 & 0.12 \\
\hline AMV059 & $08^{0} 57^{\prime} 500^{\prime \prime} \mathrm{N}$ & $07^{0} 29^{\prime} 835^{\prime \prime} \mathrm{E}$ & 0.06 & 0.11 \\
\hline AMV060 & $08^{0} 57^{\prime} 499^{\prime \prime} \mathrm{N}$ & $07^{0} 29^{\prime} 862{ }^{\prime \prime} \mathrm{E}$ & 0.06 & 0.11 \\
\hline AMV061 & $08^{0} 57^{\prime} 500^{\prime \prime} \mathrm{N}$ & $07^{0} 29^{\prime} 846^{\prime \prime} \mathrm{E}$ & 0.06 & 0.11 \\
\hline AMV062 & $08^{0} 57^{\prime} 500^{\prime \prime} \mathrm{N}$ & $07^{0} 29^{\prime} 848^{\prime \prime} \mathrm{E}$ & 0.06 & 0.11 \\
\hline AMV063 & $08^{0} 57^{\prime} 505^{\prime \prime} \mathrm{N}$ & $07^{0} 29^{\prime} 851^{\prime \prime} \mathrm{E}$ & 0.05 & 0.09 \\
\hline AMV064 & $08^{0} 57^{\prime} 502{ }^{\prime \prime} \mathrm{N}$ & $07^{0} 29^{\prime} 860$ ' $\mathrm{E}$ & 0.06 & 0.11 \\
\hline AMV065 & $08^{0} 57^{\prime} 492^{\prime \prime} \mathrm{N}$ & $07^{0} 29^{\prime} 865^{\prime \prime} \mathrm{E}$ & 0.13 & 0.23 \\
\hline AMV066 & $08^{0} 57^{\prime} 487^{\prime \prime} \mathrm{N}$ & $07^{0} 29^{\prime} 867^{\prime \prime} \mathrm{E}$ & 0.12 & 0.21 \\
\hline AMV067 & $08^{0} 57^{\prime} 494^{\prime \prime} \mathrm{N}$ & $07^{0} 29^{\prime} 869^{\prime \prime} \mathrm{E}$ & 0.08 & 0.14 \\
\hline AMV068 & $08^{0} 57^{\prime} 495^{\prime \prime} \mathrm{N}$ & $07^{0} 29^{\prime} 865^{\prime \prime} \mathrm{E}$ & 0.11 & 0.19 \\
\hline AMV069 & $08^{0} 57^{\prime} 500^{\prime \prime} \mathrm{N}$ & $07^{0} 29^{\prime} 868^{\prime \prime} \mathrm{E}$ & 0.12 & 0.21 \\
\hline AMV070 & $08^{0} 57^{\prime} 505^{\prime \prime} \mathrm{N}$ & $07^{0} 29^{\prime} 866^{\prime \prime} \mathrm{E}$ & 0.08 & 0.14 \\
\hline AMV071 & $08^{0} 57^{\prime} 508^{\prime \prime} \mathrm{N}$ & $07^{0} 29^{\prime} 880^{\prime \prime} \mathrm{E}$ & 0.05 & 0.09 \\
\hline AMV072 & $08^{0} 57^{\prime} 516^{\prime \prime} \mathrm{N}$ & $07^{0} 29^{\prime} 878^{\prime \prime} \mathrm{E}$ & 0.04 & 0.07 \\
\hline AMV073 & $08^{0} 57^{\prime} 513^{\prime \prime} \mathrm{N}$ & $07^{0} 29^{\prime} 872^{\prime \prime} \mathrm{E}$ & 0.05 & 0.09 \\
\hline AMV074 & $08^{0} 57^{\prime} 512^{\prime \prime} \mathrm{N}$ & $07^{0} 29^{\prime} 825^{\prime \prime} \mathrm{E}$ & 0.06 & 0.11 \\
\hline AMV075 & $08^{0} 57^{\prime} 5099^{\prime \prime} \mathrm{N}$ & $07^{0} 29^{\prime} 835^{\prime \prime} \mathrm{E}$ & 0.05 & 0.09 \\
\hline AMV076 & $08^{0} 57^{\prime} 637^{\prime \prime} \mathrm{N}$ & $07^{0} 29^{\prime} 788^{\prime \prime} \mathrm{E}$ & 0.05 & 0.09 \\
\hline AMV077 & $08^{0} 57^{\prime} 637^{\prime \prime} \mathrm{N}$ & $07^{0} 29^{\prime} 788^{\prime \prime} \mathrm{E}$ & 0.12 & 0.21 \\
\hline AMV078 & $08^{0} 57^{\prime} 651^{\prime \prime} \mathrm{N}$ & $07^{0} 29^{\prime} 797^{\prime \prime} \mathrm{E}$ & 0.11 & 0.19 \\
\hline AMV079 & $08^{0} 57^{\prime} 645^{\prime \prime} \mathrm{N}$ & $07^{0} 29{ }^{\prime} 766^{\prime \prime} \mathrm{E}$ & 0.14 & 0.25 \\
\hline AMV080 & $08^{0} 57^{\prime} 659^{\prime \prime} \mathrm{N}$ & $07^{0} 29^{\prime} 763$ ' $\mathrm{E}$ & 0.11 & 0.19 \\
\hline AMV081 & $08^{0} 57^{\prime} 670^{\prime \prime} \mathrm{N}$ & $07^{0} 29^{\prime} 766^{\prime \prime} \mathrm{E}$ & 0.11 & 0.19 \\
\hline AMV082 & $08^{0} 57^{\prime} 675^{\prime \prime} \mathrm{N}$ & $07^{0} 29^{\prime} 777^{\prime \prime} \mathrm{E}$ & 0.13 & 0.23 \\
\hline AMV083 & $08^{0} 57^{\prime} 672^{\prime \prime} \mathrm{N}$ & $07^{0} 29^{\prime} 789^{\prime \prime} \mathrm{E}$ & 0.07 & 0.12 \\
\hline AMV084 & $08^{0} 57^{\prime} 674^{\prime \prime} \mathrm{N}$ & $07^{0} 29^{\prime} 818^{\prime \prime} \mathrm{E}$ & 0.07 & 0.12 \\
\hline AMV085 & $08^{0} 57^{\prime} 679^{\prime \prime} \mathrm{N}$ & $07^{0} 29^{\prime} 829^{\prime \prime} \mathrm{E}$ & 0.08 & 0.14 \\
\hline AMV086 & $08^{0} 57^{\prime} 689^{\prime \prime} \mathrm{N}$ & $07^{0} 29^{\prime} 842^{\prime \prime} \mathrm{E}$ & 0.10 & 0.18 \\
\hline AMV087 & $08^{0} 57^{\prime} 697^{\prime \prime} \mathrm{N}$ & $07^{0} 29^{\prime} 843^{\prime \prime} \mathrm{E}$ & 0.09 & 0.16 \\
\hline AMV088 & $08^{0} 57^{\prime} 706^{\prime \prime} \mathrm{N}$ & $07^{0} 29^{\prime} 861^{\prime \prime} \mathrm{E}$ & 0.11 & 0.19 \\
\hline AMV089 & $08^{0} 57^{\prime} 702^{\prime \prime} \mathrm{N}$ & $07^{0} 29^{\prime} 880^{\prime \prime} \mathrm{E}$ & 0.18 & 0.32 \\
\hline AMV090 & $08^{0} 57^{\prime} 700{ }^{\prime \prime} \mathrm{N}$ & $07^{0} 29^{\prime} 898^{\prime \prime} \mathrm{E}$ & 0.15 & 0.26 \\
\hline AMV091 & $08^{0} 57^{\prime} 714^{\prime \prime} \mathrm{N}$ & $07^{0} 29^{\prime} 895^{\prime \prime} \mathrm{E}$ & 0.11 & 0.19 \\
\hline AMV092 & $08^{0} 57^{\prime} 712^{\prime \prime} \mathrm{N}$ & $07^{0} 29^{\prime} 906^{\prime \prime} \mathrm{E}$ & 0.07 & 0.12 \\
\hline AMV093 & $08^{0} 57^{\prime} 707^{\prime \prime} \mathrm{N}$ & $07^{0} 29^{\prime} 911^{\prime \prime} \mathrm{E}$ & 0.06 & 0.11 \\
\hline AMV094 & $08^{0} 57^{\prime} 705^{\prime \prime} \mathrm{N}$ & $07^{0} 29^{\prime} 916^{\prime \prime} \mathrm{E}$ & 0.14 & 0.25 \\
\hline AMV095 & $08^{0} 57^{\prime} 699^{\prime \prime} \mathrm{N}$ & $07^{0} 29^{\prime} 912{ }^{\prime \prime} \mathrm{E}$ & 0.13 & 0.23 \\
\hline AMV096 & $08^{0} 57^{\prime} 694^{\prime \prime} \mathrm{N}$ & $07^{0} 29^{\prime} 090$ ' $\mathrm{E}$ & 0.12 & 0.21 \\
\hline AMV097 & $08^{0} 57^{\prime} 689^{\prime \prime} \mathrm{N}$ & $07^{0} 29^{\prime} 899^{\prime \prime} \mathrm{E}$ & 0.08 & 0.14 \\
\hline AMV098 & $08^{0} 57^{\prime} 692^{\prime \prime} \mathrm{N}$ & $07^{0} 29^{\prime} 885^{\prime \prime} \mathrm{E}$ & 0.10 & 0.18 \\
\hline AMV099 & $08^{0} 57^{\prime} 688^{\prime \prime} \mathrm{N}$ & $07^{0} 29^{\prime} 873^{\prime \prime} \mathrm{E}$ & 0.09 & 0.16 \\
\hline AMV100 & $08^{0} 57^{\prime} 689^{\prime \prime} \mathrm{N}$ & $07^{0} 29^{\prime} 863^{\prime \prime} \mathrm{E}$ & 0.11 & 0.19 \\
\hline
\end{tabular}




\begin{tabular}{|c|c|c|c|c|}
\hline AMV101 & $08^{0} 57^{\prime} 664^{\prime \prime} \mathrm{N}$ & $07^{0} 29{ }^{\prime} 831$ 'E & 0.12 & 0.21 \\
\hline AMV102 & $08^{0} 57^{\prime} 662^{\prime \prime} \mathrm{N}$ & $07^{0} 29^{\prime} 843^{\prime \prime} \mathrm{E}$ & 0.14 & 0.25 \\
\hline AMV103 & $08^{0} 57^{\prime} 659^{\prime \prime} \mathrm{N}$ & $07^{0} 29^{\prime} 856^{\prime \prime} \mathrm{E}$ & 0.18 & 0.32 \\
\hline AMV104 & $08^{0} 57^{\prime} 655^{\prime \prime} \mathrm{N}$ & $07^{0} 29^{\prime} 655^{\prime \prime} \mathrm{E}$ & 0.16 & 0.28 \\
\hline AMV105 & $08^{0} 58^{\prime} 7299^{\prime \prime} \mathrm{N}$ & $07^{0} 29^{\prime} 820{ }^{\prime \prime} \mathrm{E}$ & 0.20 & 0.35 \\
\hline AMV106 & $08^{0} 58^{\prime} 076^{\prime \prime} \mathrm{N}$ & $07^{0} 29^{\prime} 837^{\prime \prime} \mathrm{E}$ & 0.09 & 0.16 \\
\hline AMV107 & $08^{0} 58^{\prime} 081^{\prime \prime} \mathrm{N}$ & $07^{0} 29^{\prime} 846^{\prime \prime} \mathrm{E}$ & 0.13 & 0.23 \\
\hline AMV108 & $08^{0} 58^{\prime} 082^{\prime \prime} \mathrm{N}$ & $07^{0} 29{ }^{\prime} 860^{\prime \prime} \mathrm{E}$ & 0.08 & 0.14 \\
\hline AMV109 & $08^{0} 58^{\prime} 113^{\prime \prime} \mathrm{N}$ & $07^{0} 29^{\prime} 865^{\prime \prime} \mathrm{E}$ & 0.09 & 0.16 \\
\hline AMV110 & $08^{0} 58^{\prime} 118^{\prime \prime} \mathrm{N}$ & $07^{0} 29^{\prime} 856^{\prime \prime} \mathrm{E}$ & 0.12 & 0.21 \\
\hline AMV111 & $08^{0} 58^{\prime} 116^{\prime \prime} \mathrm{N}$ & $07^{0} 29^{\prime} 827^{\prime \prime} \mathrm{E}$ & 0.12 & 0.21 \\
\hline AMV112 & $08^{0} 58^{\prime} 121{ }^{\prime \prime} \mathrm{N}$ & $07^{0} 299^{\prime} 729{ }^{\prime \prime} \mathrm{E}$ & 0.11 & 0.19 \\
\hline AMV113 & $08^{0} 58^{\prime} 131^{\prime \prime} \mathrm{N}$ & $07^{0} 29^{\prime} 809^{\prime \prime} \mathrm{E}$ & 0.08 & 0.14 \\
\hline AMV114 & $08^{0} 58^{\prime} 143^{\prime \prime} \mathrm{N}$ & $07^{0} 29^{\prime} 810^{\prime \prime} \mathrm{E}$ & 0.07 & 0.12 \\
\hline AMV115 & $08^{0} 58^{\prime} 154^{\prime \prime} \mathrm{N}$ & $07^{0} 29^{\prime} 817^{\prime \prime} \mathrm{E}$ & 0.05 & 0.09 \\
\hline AMV116 & $08^{0} 58^{\prime} 162^{\prime \prime} \mathrm{N}$ & $07^{0} 29^{\prime} 815^{\prime \prime} \mathrm{E}$ & 0.06 & 0.11 \\
\hline AMV117 & $08^{0} 58^{\prime} 176^{\prime \prime} \mathrm{N}$ & $07^{0} 29^{\prime} 823^{\prime \prime} \mathrm{E}$ & 0.08 & 0.14 \\
\hline AMV118 & $08^{0} 58^{\prime} 180^{\prime \prime} \mathrm{N}$ & $07^{0} 29^{\prime} 837^{\prime \prime} \mathrm{E}$ & 0.12 & 0.21 \\
\hline AMV119 & $08^{0} 58^{\prime} 174^{\prime \prime} \mathrm{N}$ & $07^{0} 29^{\prime} 8599^{\prime \prime} \mathrm{E}$ & 0.08 & 0.14 \\
\hline AMV120 & $08^{0} 58^{\prime} 186^{\prime \prime} \mathrm{N}$ & $07^{0} 29^{\prime} 869^{\prime \prime} \mathrm{E}$ & 0.04 & 0.07 \\
\hline AMV121 & $08^{0} 58^{\prime} 121^{\prime \prime} \mathrm{N}$ & $07^{0} 29^{\prime} 835^{\prime \prime} \mathrm{E}$ & 0.14 & 0.25 \\
\hline AMV122 & $08^{0} 58^{\prime} 081^{\prime \prime} \mathrm{N}$ & $07^{0} 29^{\prime} 809{ }^{\prime \prime} \mathrm{E}$ & 0.08 & 0.14 \\
\hline AMV123 & $08^{0} 58^{\prime} 089^{\prime \prime} \mathrm{N}$ & $07^{0} 29^{\prime} 812^{\prime \prime} \mathrm{E}$ & 0.13 & 0.23 \\
\hline AMV124 & $08^{0} 58^{\prime} 508^{\prime \prime} \mathrm{N}$ & $07^{0} 29^{\prime} 817^{\prime \prime} \mathrm{E}$ & 0.12 & 0.21 \\
\hline AMV125 & $08^{0} 58^{\prime} 480^{\prime \prime} \mathrm{N}$ & $07^{0} 29^{\prime} 806^{\prime \prime} \mathrm{E}$ & 0.06 & 0.11 \\
\hline AMV126 & $08^{0} 58^{\prime} 443^{\prime \prime} \mathrm{N}$ & $07^{0} 29^{\prime} 819{ }^{\prime \prime} \mathrm{E}$ & 0.06 & 0.11 \\
\hline AMV127 & $08^{0} 58^{\prime} 424^{\prime \prime} \mathrm{N}$ & $07^{0} 29^{\prime} 828^{\prime \prime} \mathrm{E}$ & 0.07 & 0.12 \\
\hline AMV128 & $08^{0} 58^{\prime} 417^{\prime \prime} \mathrm{N}$ & $07^{0} 29^{\prime} 827^{\prime \prime} \mathrm{E}$ & 0.12 & 0.21 \\
\hline AMV129 & $08^{0} 58^{\prime} 408^{\prime \prime} \mathrm{N}$ & $07^{0} 29$ '823' 'Е & 0.10 & 0.18 \\
\hline AMV130 & $08^{0} 58^{\prime} 408^{\prime \prime} \mathrm{N}$ & $07^{0} 29^{\prime} 810^{\prime \prime} \mathrm{E}$ & 0.14 & 0.25 \\
\hline AMV131 & $08^{0} 58^{\prime} 418^{\prime \prime} \mathrm{N}$ & $07^{0} 29^{\prime} 805^{\prime \prime} \mathrm{E}$ & 0.10 & 0.18 \\
\hline AMV132 & $08^{0} 58^{\prime} 430^{\prime \prime} \mathrm{N}$ & $07^{0} 29^{\prime} 798^{\prime \prime} \mathrm{E}$ & 0.08 & 0.14 \\
\hline \multirow[t]{2}{*}{ AMV133 } & $08^{0} 58^{\prime} 479^{\prime \prime} \mathrm{N}$ & $07^{0} 29^{\prime} 789^{\prime \prime} \mathrm{E}$ & 0.12 & 0.21 \\
\hline & & Mean \pm SD & $0.10 \pm 0.03$ & $0.20 \pm 0.06$ \\
\hline
\end{tabular}

From the results of the measurements presented in Table 1, location AMV013 has the highest annual effective dose rate of $0.39 \mathrm{mSv} / \mathrm{yr}$. Locations AMV105, AMV012, AMV089 and AMV103 have annual effective dose rate of $0.35 \mathrm{mSv} / \mathrm{yr}, 0.32$ $\mathrm{mSv} / \mathrm{yr}, 0.32 \mathrm{mSv} / \mathrm{yr}$ and $0.32 \mathrm{mSv} / \mathrm{yr}$ respectively. Locations AMV072 and AMV120 have the least annual effective dose rate of $0.04 \mathrm{mSv} / \mathrm{yr}$. However, these values are seen to be lower than the recommended value of $1.0 \mathrm{mSv} / \mathrm{yr}$ for public exposure placed by the Basic Safety Standards (BSS) SCHEDULE II and the International Commission on Radiological Protection (ICRP) REPORT 60 (ICRP, 1991). This indicates that the automobile mechanics and the people working and living around the area are not exposed to high doses of radiation as a result of the activities taken place in the mechanic village.

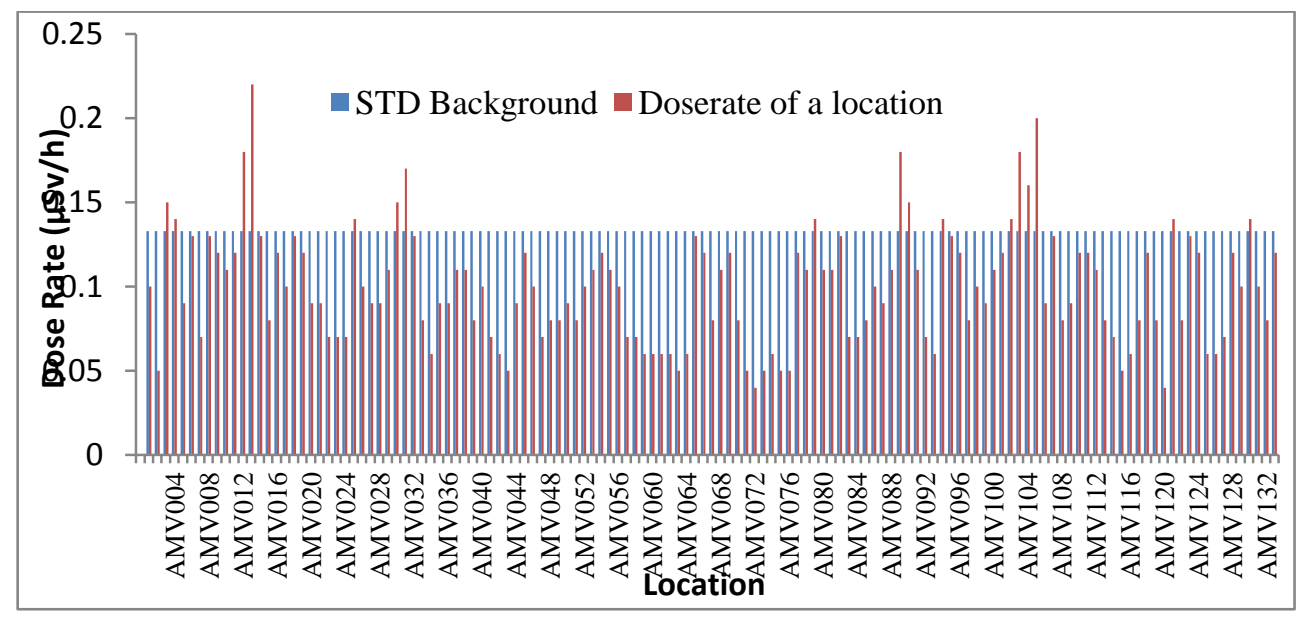

Fig 3: Average Equivalent Dose Rate of different location

Conclusion: The environmental monitoring of radiation dose rates has been computed in and around the Apo Automobile Mechanic Village, Abuja using in-situ measurement method. This work revealed that the average dose equivalent varied from $0.04 \mu \mathrm{Sv} / \mathrm{h}$ to $0.22 \mu \mathrm{Sv} / \mathrm{h}$ with a mean of $0.10 \pm 0.03 \mu \mathrm{Sv} / \mathrm{h}$ which is below the standard background radiation of 0.133 $\mu \mathrm{Sv} / \mathrm{h}$. The study also revealed that the average annual effective dose rate is approximately $0.20 \pm 0.06$ $\mathrm{mSv} / \mathrm{yr}$ which is lower than the value of $1.0 \mathrm{mSv} / \mathrm{yr}$ 
averaged over five consecutive years according to the dose limit recommended by the Basic Safety Standards (BSS) SCHEDULE II and the International Commission on Radiological Protection (ICRP) REPORT 60. This indicates that the automobile technicians, craftsmen and the people living and working within the area are safe and are not exposed to high doses of radiation as a result of activities in the Apo Automobile Mechanic Village. The results from this work will form the baseline data which will be useful in assessing contribution to radiation in the environment from future activities of the Automobile Mechanic Village.

\section{REFERENCES}

A Sadiq, E. H Agba (2011). Background Radiation in Akwanga, Nigeria. FACTA UNIVERSITATIS Series: Working and Living Environmental Protection Vol. 8, No 1, pp. 7 - 11.

Angela C. Udebuani, Chidiogo G. Okoli, Ifeanyi C. Okoli, Harriet C. Nwigwe and Patrick T. E. Ozoh (2011). Assessments of the volume and disposal methods of spent engine oil generated in Nekede mechanic village, Owerri, Nigeria. Report and Opinion 3(2):31-36

E. Howdershelt (2000). Radioactive Scrap Metal. Abintra Press

G. O. Avwiri, P. I. Enyinna and E. O. Agbalagba (2010). Occupational Radiation Levels in Solid Mineral Producing Areas of Abia State, Nigeria. Scientia Africana, Vol. 9 (No.1), pp 93-97.

ICRP (1991): Recommendations of the International Commission on Radiological Protection. ICRP Publication no. 60. Oxford: Pergamon press.

Lenka M and Peter E (2010). End-of-waste criteria for iron and steel scrap: technical proposals. EUR-scientific and technical research seriesISSN 10185593

M. Amiri, R. Abdi, A. Shabestani Monfared (2011). Estimation of external natural background gamma ray doses to the population of Caspian coastal provinces in North of Iran. Iran. J. Radiat. Res., 9(3): 183-186

MDH: Environment Radiation Monitoring (2008). Minnesota Department of Health, Radiation Control Unit, 651-201-4545.
Muhammad A. M. Idris I. F. Simon P. M., and Abdullahi S. A. (2011). Determination of Absorbed and Effective Dose from Natural Background Radiation around a Nuclear Research Facility. American Journal of Environmental Sciences 7 (2): 173-177

O. D. Nworgu, O. D. Osahon, F.E. Franca (2012). Measurement of Gamma Radiation in Automobile Mechanic Workshops in an Area of Benin City, Nigeria. Advanced Materials Research Vol. 367 pp 801-805

O. D. Oyeyinka, I. U. James, E. C. Akueche, O. A. Shonowo, C. A. Adesanmi (2012). Estimation of Radiation Dose Rate Levels around a Nuclear Establishment in Abuja, North Central, Nigeria. Science and Technology 2(6): 163-167

Office of Radiation Protection, 2008. Division of Environmental Health, Washington State Department of Health

Olarinoye, I. O; Sharifat, I; Baba-Kutigi, A. N; Kolo, M. T; Aladeniyi, K. (2010). Measurement of Background gamma radiation levels at two Tertiary Institutions in Minna, Nigeria. J. App. Sc. Environ. Management, Vol. 14 (1): 5962.

Tayyeb Allahverdi Pourfallah, Hamed Babapour, Maryam Shahidi (2012). Evaluation of High Level Environmental Background Radiation Areas and its Variation in Ramsar. Iranian Journal of Medical Physics Vol. 9, No. 2, 87-92

Tchokossa, P; Olomo, J. B; Balogun, F. A; Adesanmi, C. A; (2012) Radiological Study of Soils in Oil and Gas Producing Areas in Delta State, Nigeria. Radiat. Prot. Dos. Pp 1-6

UNSCEAR (2000). United Nation Scientific Committee on the Effects of Atomic Radiation. Sources and effect of Ionizing radiation. Report to the general assembly with scientific annexes. United Nations; New York 